\title{
Trellis Codes with Low Ones Density for the OR Multiple Access Channel
}

\author{
M. Griot, A. I. Vila Casado, W.-Y. Weng, H. Chan, J. Basak, E. Yablonovitch, I. Verbauwhede, B. Jalali, and R. D. Wesel \\ Dept. of Electrical Engineering, University of California \\ Los Angeles, CA 90095 \\ \{mgriot,avila,wenyen,herwin,juthika,eliy,ingrid,jalali,wesel\}@ee.ucla.edu
}

\begin{abstract}
This paper presents trellis codes for the $\mathbf{Z}$ channel designed to maintain a relatively low ones density. These codes have applications in pulse-position modulation systems and as a solution for uncoordinated communication on the binary OR multiple-access channel (MAC). In this paper we consider the latter application to demonstrate the performance of the codes.

The OR channel provides an unusual opportunity where single-user decoding permits operation at about $70 \%$ of the full multiple-access channel sum capacity. The interleaver-division multiple access technique applied in this paper should approach that performance with turbo solutions. However, the current paper focuses on very low latency codes with simple decoding, intended for very high speed (gigabits per second) applications. Namely, it focuses on nonlinear trellis codes that provide about $30 \%$ of the full multiple-access sum capacity at high speeds and with very low latency. These trellis codes are designed specifically for the Z-Channel that arises in a multiple-user OR channel, when the other users are treated as noise. In order to optimize the sum-capacity of the OR-MAC, the trellis code transmits codewords with a ones density much less than 50\%. Also, a union bound technique that predicts the performance of these codes is presented. Results from simulations and a working FPGA implementation are shown.
\end{abstract}

\section{INTRODUCTION}

Completely uncoordinated transmission on the OR-MAC is theoretically possible with the same efficiency as TDMA if joint decoding is employed. Joint decoding can be simplified to sequential decoding if the ones densities of each transmitter are carefully controlled [1], but this level of coordination is not qualitatively different from assigning time slots.

These schemes are fully efficient in that each received bit conveys one useful bit of information. However, completely uncoordinated transmissions using interleaver-division multiple access (IDMA)[2][3] and simple decoding that treats other users as noise is an attractive alternative. Surprisingly, in the multiple-user OR channel, this relatively low-complexity approach can theoretically achieve about $70 \%$ of full efficiency.

This paper presents an uncoordinated multiple access system employing IDMA in which the other users are treated as noise. To allow decoding at very high speeds in the near future, this paper investigates trellis codes which operate in a range of $30 \%$ of full efficiency. In other work [13], we are also designing turbo solutions, which approach the $70 \%$ limit with higher complexity and latency.

This work was supported by the Defence Advanced Research Project Agency under SPAWAR Systems Center San Diego Grant N66001-02-1-8938.
Section II reviews uncoordinated multiple access in the OR channel. Section III presents the design of nonlinear trellis coded modulation (NL-TCM) for this application. Section IV presents a transfer-function bound for NL-TCMs operating on the Z-Channel. Section V presents performance results, and Section VI concludes the paper.

\section{UnCOORdinAted Multiple ACCESS IN THE OR CHANNEL}

In the multiple access OR channel, if all users transmit a zero, then the channel output is a zero. However, if even one user transmits a one, then the channel output is a one. The information-theoretic capacity region of this channel is the union of all rate pairs with a sum-rate (the sum of all the rates of the system) less than 1 . As discussed above, this capacity may be achieved with time-division multiple access, joint decoding of all the transmitted sequences, or sequential decoding if transmitted ones densities are carefully controlled. All of these solutions require either coordination of all users or a very complex decoder, neither of which is currently feasible at gigabit per second sum rates.

In high-rate applications where complete coordination is not possible and joint or successive decoding is unavailable for complexity reasons, the other users must be treated as noise. This transforms the OR channel into the Z-Channel shown in Fig. 1. If we assume that all users have the same transmitted ones density, the transition probability is a function of the same transmitted ones density employed by the desired user. Though not required, we maintain this assumption throughout the paper for simplicity.

The maximum theoretical sum-rate when treating the other users as noise (assuming each user employs the same ones density) decreases as the number of users increases. However, it is always lower bounded by $\ln 2 \simeq 0.6931$. This is a relatively small loss in rate for the substantial reduction in complexity. In order to be able to achieve this maximum theoretical sum-rate, the optimal ones density of each individual user decreases as the number of users increase. For example, the optimal density of ones is $p_{1} \simeq 0.2864$ for 2 equal-rate users, $p_{1} \simeq 0.1080$ for 6 equal-rate users, and $p_{1} \simeq 0.0558$ for 12 equal-rate users.

On the other hand, when maintaining equally likely ones and zeros $\left(p_{1}=0.5\right)$ the maximum theoretical sum-rate rapidly decreases to zero with the number of users. For example, the maximum achievable sum-rate is less than $10 \%$ for 6 users and 


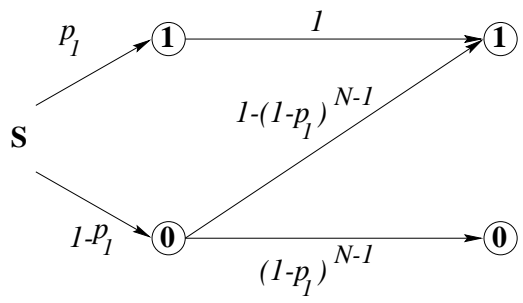

Fig. 1. Z-channel resulting from the OR-MAC channel when other users are treated as noise.

less than $1 \%$ for 10 users. Poor performance of the $p_{1}=0.5$ case demonstrates that codes with low ones densities are a requirement for this application.

One successful approach for uncoordinated multiple-access is Interleaver-Division Multiple-Access (IDMA) [2][3]. With IDMA, every user has the same channel code, but each user's code bits are permuted using a randomly drawn interleaver, unique with extremely high probability. The receiver is assumed to know the interleaver of the desired user. With IDMA in the OR-MAC, a receiver sees the desired signal corrupted by a memoryless Z-channel. We performed simulations comparing an NL-TCM code under two channels: 1) a 6-user ORMAC channel using IDMA and 2) the equivalent Z-channel that the receiver would see if the errors were not generated by codewords but by random errors. The performance was the same. Thus, in the context of IDMA, the remaining challenge is the design of a good code with the desired ones density.

\section{NL-TCM With CONTROLLED ONES DENSITY}

Papers appearing since the 1950's have addressed the problem of designing codes with $p_{1}=0.5$ for the Z-channel. See [4] for a unified account on such codes and [5] for the most recent advances in this field. Only recently there has been work on LDPC codes with an arbitrary density of ones, see [6] and [7]. This manuscript is the first to our knowledge to address the design of trellis codes with an arbitrary density of ones for the Z-Channel.

In this section, we present a design technique for trellis codes for the Z-channel with an arbitrary ones density $p_{1}$. Our goal is to maximize the minimum directional Hamming distance (a metric we'll define below) between codewords, and the rates considered will be of the form $1 / N$. We use a conventional feed-forward encoder in order to determine the branches of the trellis, but instead of using generator polynomials to compute the output of each branch, a nonlinear table-lookup will directly assign the output values.

\section{A. Directional Hamming Distance}

In the Z-Channel, a transmitted 1 will always induce a received 1 . Thus, to make a decoding error, the decoder must see ones in all the bit positions where the incorrect codeword has ones. Let us define the directional Hamming distance $d_{D}\left(c_{1}, c_{2}\right)$ between two codewords $c_{1}$ and $c_{2}$ as the number of positions at which $c_{1}$ has a 0 and $c_{2}$ a 1 .

Consider the Z-Channel with a probability of 0-to-1 transition less than 0.5 . If two codewords $c_{1}$ and $c_{2}$ have different
Hamming weights, the codeword with the smaller Hamming weight (denote it $c_{2}$ ) will never be incorrectly decoded by a maximum likelihood (ML) decoder when the code with the larger Hamming weight $\left(c_{1}\right)$ is transmitted. Then, when considering the pairwise behavior of these two codewords, the only distance that matters is $d_{D}\left(c_{2}, c_{1}\right)$. On the other hand, if both codewords have the same Hamming weight, the directional Hamming distances are equal and errors can be made in either direction. Note that in any case, the directional distance that matters is the larger of the two.

Thus a proper definition for the pairwise design metric should be the maximum pairwise directional Hamming distance:

$$
d\left(c_{i}, c_{j}\right)=d\left(c_{j}, c_{i}\right)=\max \left[d_{D}\left(c_{i}, c_{j}\right), d_{D}\left(c_{j}, c_{i}\right)\right]
$$

This metric for the Z-Channel is well known, appearing in [4] and [5] among other papers.

\section{B. Pessimistic definition of distance}

Due to its non-linearity, this definition of distance cannot be applied branch-wise, since it is impossible to tell from an individual branch which codeword will end up having more Hamming weight. For that reason, we will use a pessimistic definition of distance for our trellis code design, considering both directional distances. Namely, the safest definition of branch-wise distance between any two branches $b_{i}$ and $b_{j}$ would be

$$
d_{p}=\min \left[d_{D}\left(b_{i}, b_{j}\right), d_{D}\left(b_{j}, b_{i}\right)\right],
$$

which is the pessimistic branch-wise metric that will be maximized in our design.

With this branch-wise metric, codewords with equal Hamming weights produce larger values of $d_{p}$ than codewords with different Hamming weights, so we will assign output values to the trellis branches with as similar Hamming weight as possible, preferably equal.

\section{NL-TCM Code Design}

As we mentioned before, the code design consists of assigning output values to the branches of the trellis. Those outputs have to maintain the desired average density of ones $p_{1}$. We will present an extension to the Ungerboeck's rules [8] intended to maximize the minimum pessimistic distance $d_{\min }$ using the pessimistic branch-wise metric introduced in section (III-B).

1) Choosing the Hamming weight of the branches: The first step in the design is to assign Hamming weights $\left(W_{h}\right)$ to each of the branches. For a rate of $1 / N$, using a $2^{\nu}$-state encoder ( $B=2^{\nu+1}$ branches), and an optimal ones density of $p_{1}$, there should be $B_{s}$ branches with Hamming weight $s=$ floor $\left(p_{1} \cdot N\right)$ and $B_{s+1}=B-B_{s}$ branches with Hamming weight $s+1$, where $B_{s}$ should be chosen to minimize the deviation from the desired ones density:

$$
\text { deviation }=\left|p_{1} \cdot N-\left(B_{s+1} \cdot(s+1)+B_{s} \cdot s\right) / B\right| .
$$




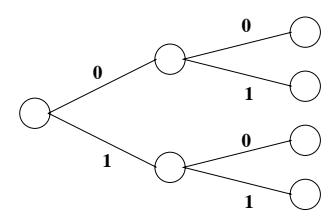

(a)

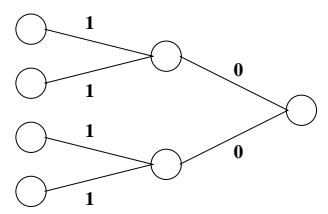

Fig. 2. (a) Four paths emanating from the same state in two trellis sections (b) Four paths merging into the same state in two trellis sections. Branches are labeled with the input bits that induce traversal of the branch.

2) Choosing all branches to have distance of at least 1 between each other: If possible, it would be desirable to have all branches have a pessimistic distance $\left(d_{p}\right)$ of at least 1 between each other.

When all branches have the same weight $W_{H}=s$, there are $\left(\begin{array}{c}N \\ s\end{array}\right)$ branch labels with $d_{p} \geq 1$ between each other. For example, for $N=17$ and $p_{1}=1 / 8$, and choosing all branches to have $W_{H}=2$, we can have $\left(\begin{array}{c}17 \\ 2\end{array}\right)=134$ different branches, so if we wanted to have all branch labels to be different so that $d_{p} \geq 1$ always, we could use a trellis with 64 states (represented by 6 bits) and 128 branches. The minimum number of branches that two paths can differ in a $2^{\nu}$-state encoder is $\nu+1$. Thus, if each section adds at least 1 to the pessimistic distance, then $d_{\min } \geq \nu+1$.

If repeated output values are necessary, often some branches can be strategically chosen to be equal while still maintaining $d_{\min } \geq \nu+1$.

3) Ungerboeck's rule: Once we choose the weights of the branches, and the possible output values that will make all branches different (or almost all, allowing some to be equal as explained in the previous section), we have to assign output values to branches. Our main approach for this assignment is to apply Ungerboeck's idea of maximizing the distance between splits and merges [8]. The Hamming weights of the branches are at least $s$. In the application considered in this work, where low rate codes with low ones densities are required, all splits and merges can be chosen to have a pessimistic distance of at least $s$ between each other. Thus, applying Ungerboeck's rule a $d_{\min } \geq 2 s+\nu-1$ can be achieved.

4) Extending Ungerboeck's rule into the trellis: One can extend Ungerboeck's rule more deeply into the trellis, and maximize not only the distance between splits, and the distance between merges, but the distance between the 4 branches emanating from a split in the previous trellis section, or the 8 branches emanating from a split two sections before, and so on. One can do the same with the merges moving backwards in the trellis. Notice that by maximizing the distance between the 8 branches emanating from a split two sections before, we are also maximizing the distance between all 4 branches emanating from a split a trellis section before, and all splits. The same idea applies to the merges. If we consider $h$ sections after a split, and $g$ sections before a merge, the new bound for the minimum distance is $d_{\min } \geq s \cdot(h+g)+\nu+1-(h+g)$. Fig. 2 shows the trellis branches involved with $\mathrm{h}=2$ and $\mathrm{g}=2$.

The largest possible values of $h$ and $g$ are given by the maximum number of output values that can have maximum distance between each other. Let us explain this in more detail.

First, compute the number of branches that need to have maximum distance between each other to cover $h$ sections after a split and $g$ sections before a merge. From the splitting point of view, there will be groups of $2^{h}$ branches that need to have maximum distance between each other. From the merging point of view, there will be groups of $2^{g}$. Each branch belongs to one group of $2^{h}$ and one group of $2^{g}$, so each branch has to have maximum pessimistic distance with $2^{h}+2^{g}-2$ other branches.

Second, compute the maximum possible number of branches of maximum pessimistic distance between each other. Using the example where $N=17$ and all the branches have Hamming weight $s=2$, it is possible to have floor $\left(\frac{N}{s}\right)=8$ branches with maximum distance between each other. Let us denote this number as $T$.

Then, the constraints are (1) $2^{h} \leq T$, (2) $2^{g} \leq T$ and (3) each branch has to belong to one group of $2^{h}$ and one group of $2^{g}$. If one chooses $h$ and $g$ such that $2^{h}+2^{g}-2 \leq T$, all 3 constraints can be satisfied.

5) Designing for a very low target ones density: If the optimal ones density $p_{1} \leq 1 / 2^{\nu+1}$, all the branches can be chosen to have maximum distance between each other (we can choose the branch labels so that for any particular position of the output, there is at most 1 branch that has a 1 in that position).

For such low densities of ones, the design becomes straightforward. Compute the Hamming weight of each branch as explained in section III-C.1, and for each branch, add ones in positions that aren't used in previous branches.

\section{TRANSFER Function Bound FOR NL-TCM CODES}

Ellingsen [9] provided a combinatorial expression for an upper bound on the BER of linear block codes over the Z-channel under ML decoding. For convolutional codes assuming binary PAM or QPSK, Viterbi [10] introduced an analytical technique using generating functions to provide a union bound on the BER of convolutional codes. The technique is based on a $2^{\nu}$ state diagram for the convolutional encoder. In the case of general trellis codes where high level constellations introduce nonlinearity, Biglieri [11][12] generalized Viterbi's algorithm by using the product state diagram with $2^{2 \nu}$-states. Biglieri's algorithm can be applied to non-linear trellis codes over the Z-channel with modifications on the pairwise error probability measure.

Let $\alpha$ be the probability of a zero-to-one transition in the $\mathrm{Z}$ channel. Consider the whole binary transmitted sequence, $X^{n}$, and any other valid codeword, $\hat{X}^{n}$. The pairwise error probability of decoding $X^{n}$ into $\hat{X}^{n}$ under ML decoding is

$$
\begin{gathered}
P_{e}\left(X^{n} \rightarrow \hat{X}^{n}\right)= \\
\left\{\begin{aligned}
\frac{1}{2} \cdot \alpha^{d_{D}\left(X^{n}, \hat{X}^{n}\right)} & , W_{H}\left(X^{n}\right)=W_{H}\left(\hat{X}^{n}\right) \\
\alpha^{d_{D}\left(X^{n}, \hat{X}^{n}\right)} & , W_{H}\left(X^{n}\right)<W_{H}\left(\hat{X}^{n}\right) \\
0 & , W_{H}\left(X^{n}\right)>W_{H}\left(\hat{X}^{n}\right)
\end{aligned}\right.
\end{gathered}
$$


where $d_{D}(\cdot, \cdot)$ denotes the directional distance. If we consider the sequence pair $\left(X^{n}, \hat{X}^{n}\right)$, the error probability of transmitting one sequence and decoding the other is

$$
\begin{aligned}
& P_{e}\left(X^{n} \rightarrow \hat{X}^{n}\right)+P_{e}\left(\hat{X}^{n} \rightarrow X^{n}\right) \\
& =\alpha^{\max \left(d_{D}\left(X^{n}, \hat{X}^{n}\right), d_{D}\left(\hat{X}^{n}, X^{n}\right)\right)} \\
& \leq \frac{1}{2}\left[\alpha^{d_{D}\left(X^{n}, \hat{X}^{n}\right)}+\alpha^{d_{D}\left(\hat{X}^{n}, X^{n}\right)}\right]
\end{aligned}
$$

Therefore, if $P_{e}\left(X^{n} \rightarrow \hat{X}^{n}\right)$ is replaced (not always upperbounded) by $\frac{1}{2} \alpha^{d_{D}\left(X^{n}, \hat{X}^{n}\right)}$ for all the codewords $X^{n}$ and $\hat{X}^{n}$, the transfer function bound technique can be readily applied to the NL-TCM to yield a valid upper bound because of the additive property of the directional distance.

As in [11], the product state diagram consists of state pairs, $\left(s_{e}, s_{r}\right)$, where $s_{e}$ is the encoder state and $s_{r}$ the receiver state. Following Biglieri's notation, the product states can be divided into two sets, the good states denoted by $S_{G}$ and the bad states denoted by $S_{B}$ defined as

$$
S_{G}=\left\{\left(s_{e}, s_{r}\right) \mid s_{e}=s_{r}\right\}, S_{B}=\left\{\left(s_{e}, s_{r}\right) \mid s_{e} \neq s_{r}\right\}
$$

By suitably renumbering the product states, we get the transition matrix

$$
S(W, I)=\left[\begin{array}{l|l}
S_{G G}(W, I) & S_{G B}(W, I) \\
\hline S_{B G}(W, I) & S_{B B}(W, I)
\end{array}\right]
$$

Where the $N \times N$ matrix $S_{G G}(W, I)$ accounts for the transitions between good product states, the $N \times\left(N^{2}-N\right)$ matrix $S_{G B}(W, I)$ accounts for the transition from good product states to bad product states, and so forth. $N$ is the number of encoder states $2^{\nu}$. For each transition in the product state diagram, $S_{1} \rightarrow S_{2}$, the branch is labeled by

$$
p\left(S_{1} \rightarrow S_{2}\right) W^{d_{D}\left(x_{e}, x_{r}\right)} I^{d_{H}\left(u_{e}, u_{r}\right)}
$$

where $u_{e}$ and $x_{e}$ denote the input and output word for the encoder states respectively. Similar for the received states. $d_{H}(\cdot, \cdot)$ denotes the Hamming distance. Then the transfer function $T(W, I)$ becomes

$$
T(W, I)=p_{s}\left\{S_{G G}+S_{G B}\left(I-S_{B B}\right)^{-1} S_{B G}\right\} \mathbf{1}
$$

where $p_{s}=\left[\frac{1}{N} \frac{1}{N} \cdots \frac{1}{N}\right]$ is the probability distribution of the encoder states and $\mathbf{1}=[11 \cdots 1]^{T}$. The BER bound is computed as

$$
B E R \leq\left.\frac{1}{2} \cdot \frac{1}{k} \cdot \frac{\partial T(W, I)}{\partial I}\right|_{W=\alpha, I=1}
$$

\section{Performance Results}

We have tested the NL-TCM performance in an uncoordinated OR multiple access channel, where every user treats the others as noise, and all users transmit with the same density of ones $p_{1}$, as explained in Section II.

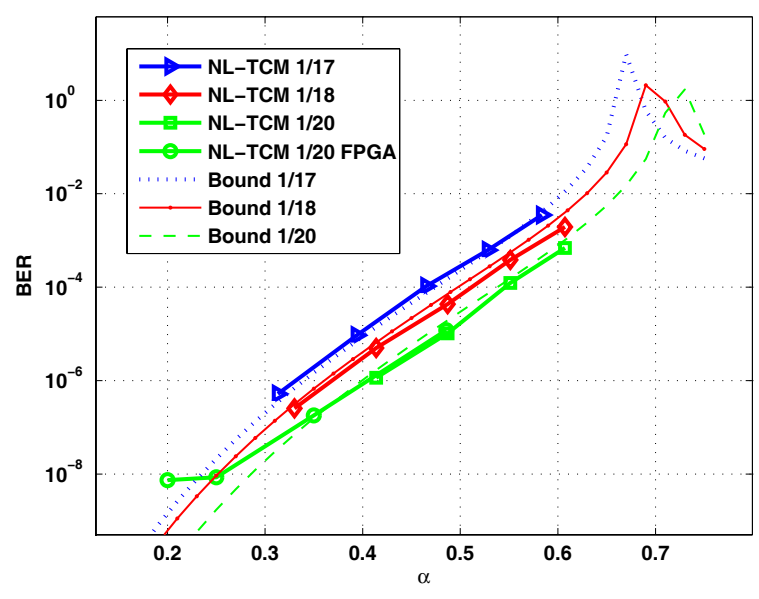

Fig. 3. BER of NL-TCM codes versus crossover probability $(\alpha)$

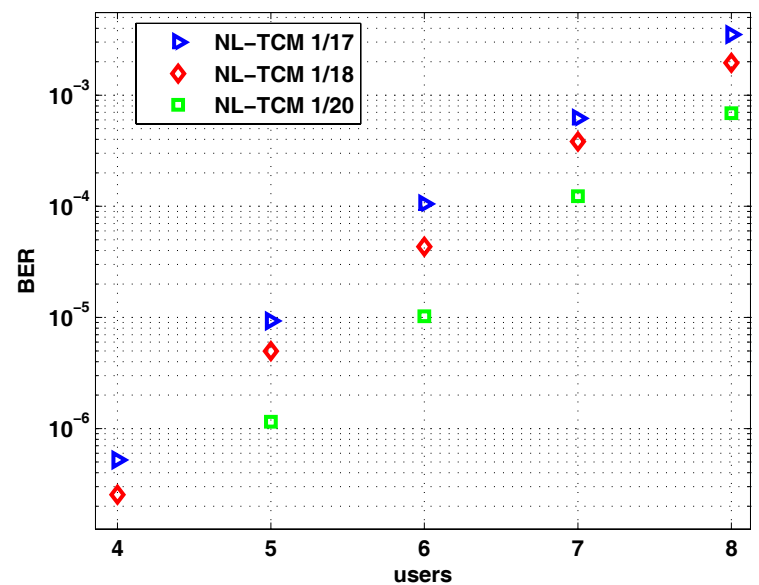

Fig. 4. BER of NL-TCM codes vs. number of users

\section{A. NL-TCM for 6-user OR-MAC}

Fig. 3 shows the BER of various 64-state NL-TCM codes designed to work in a 6-user OR-MAC channel, along with their theoretical transfer function bounds. The densities of ones are close to 0.108 , the density needed to achieve capacity for a 6-users OR-MAC when treating other users as noise. Simulations have been programmed in $\mathrm{C}$ for rate-1/17 NLTCM code with $p_{1}=2 / 17$, rate-1/18 NL-TCM code with $p_{1}=1 / 8$ and rate- $1 / 20$ NL-TCM code with $p_{1}=1 / 8$.

It can be observed that the transfer function bounds are tight in all three cases. The transfer function bound is an upper bound on the expectation assuming an infinite decoder depth, and it is not unexpected for these simulations to be slightly above the bound, since the margin is within the usual variation of a simulation around the expectation.

Also, in order to prove that NL-TCM codes are feasible today for very high speeds (optical speeds), a hardware simulation engine was built on the Xilinx Virtex2-Pro 2V20 FPGA. The simulator implemented on this device had equivalent gate 
TABLE I

BER OF NL-TCM FOR 100-USER OR-MAC

\begin{tabular}{|c|c|c|c|c|}
\hline Rate & Sum-rate & $p_{1}$ & $\alpha$ & BER \\
\hline $1 / 334$ & 0.2994 & 0.006736 & 0.48787 & $1.10 \cdot 10^{-5}$ \\
\hline $1 / 360$ & 0.2778 & 0.006944 & 0.49837 & $4.54 \cdot 10^{-6}$ \\
\hline $1 / 400$ & 0.25 & 0.006875 & 0.49489 & $9.45 \cdot 10^{-7}$ \\
\hline
\end{tabular}

count of $360 \mathrm{~K}$ gates and is able to simulate the rate $1 / 20$ code at $70 \mathrm{Mbps}$. This implementation received first prize at the 2006 student design contest sponsored by the ACM Design Automation Conference and the IEEE International Solid State Circuits Conference.

Results for rate-1/20 NL-TCM code obtained in the FPGA testbed are also shown in Fig.3. Due to design constraints, the hardware Viterbi decoder has implementation differences comparing to the software simulation. The two main differences are the traceback depth and the maximum path metric. Both of these values are set to infinity for software simulation. The hardware implementation has a traceback depth of 35 and a maximum path distance metric of 20 . These differences cause the deviation from the theoretical bound at low bit error rates.

Finally, Fig. 4 shows the BER of these codes in terms of the number of users present in an OR-MAC.

\section{B. NL-TCM for 100-user OR-MAC}

As explained in section III-C.5, the design of NL-TCM codes becomes straight-forward for low enough density of ones. As an example, we have designed NL-TCM codes for the 100-user OR-MAC case. In that case, the optimal $p_{1} \simeq 0.00691$. We designed codes for rates $1 / 400$ and $1 / 360$. Results are shown in Table I.

\section{Concatenation of NL-TCM code with a Block Code}

In applications where a very low BER is required, the rate of the NL-TCM channel code would have to be very low. A better solution is to include a high-rate block code that can correct a small number of symbol errors as an outer code, dramatically lowering the BER.

A concatenation of the rate- $1 / 20$ NL-TCM code with a (255-bytes, 247-bytes) Reed-Solomon code has been tested for the 6-user OR-MAC scenario. The rate of this code is $(247 / 255) \cdot(1 / 20) \simeq 0.0484$. Results are shown in Table II. In this example, we are achieving almost $30 \%$ of full capacity, with a BER on the order of $10^{-10}$. As an example, the OR channel can be used as a simple communications model that describes the multiple-user local area network optical channel with non-coherent combining. With the concatenation of NLTCM codes with a Reed Solomon Code we are achieving a good part of the capacity (taking into account the very low latency of the code), with a suitable BER for optics, and a feasible complexity for today's technology at optical speeds.

\section{CONCLUSIONS}

This paper addressed the problem of designing codes for the Z-channel along with an IDMA-based architecture that allows uncoordinated multiple access in the OR-MAC. In this
TABLE II

BER OF RS+NL-TCM FOR 6-USER OR-MAC

\begin{tabular}{|c|c|c|c|c|}
\hline Rate & Sum-rate & $p_{1}$ & $\alpha$ & BER \\
\hline 0.0484 & 0.29 & 0.125 & 0.4652 & $2.48 \cdot 10^{-10}$ \\
\hline
\end{tabular}

architecture, the same code is used by every user, each of which randomly picks an interleaver to permute its coded bits. For each user, every other user is treated as noise, in which case it's receiver 'sees' a Z-channel. We have addressed the problem of designing codes for this channel. These codes are required to have a relatively low ones density, requiring non-linear codes. In this work, we also required low decoder complexity to be computationally feasible at very high speeds today.

Non-linear trellis codes satisfy both requirements. A design criteria for NL-TCM codes was introduced, and tight analytical bounds on their performance over the Z-Channel, were computed. Furthermore, by concatenating these codes with highrate block codes we can achieve a good part of the capacity of the channel with a very low BER and a very fast decoder. An important feature of this solution is that the achieved sum-rate remains basically unchanged as the number of users increases as shown in section $\mathrm{V}$ for the 6-user and 100-user case. This result makes this solution especially attractive for a large number of users, where coordination becomes an issue.

\section{REFERENCES}

[1] A. Grant, B. Rimoldi, R. Urbanke, and P. Whiting. Rate-Splitting Multiple Access for Discrete Memoryless Channels. IEEE Trans. on Inform. Theory, 47(3):873-890, Mar. 2001.

[2] Li Ping, K. Y. Wu, and L. Liu. A Simple, Unified Approach to Nearly Optimal Multiuser Detection and Space-time Coding. In ITW 2002, India, October 2002.

[3] Li Ping, Lihai Liu, and W. K. Leung. A Simple Approach to NearOptimal Multiuser Detection: Interleaver-Division Multiple-Access. In IEEE Wireless Comm. and Networking Conference, pages 391-396, 2003.

[4] T. Kløve. Error correcting codes for the asymmetric channel. In Dept. Mathematics, Univ. Bergen, Bergen, Norway, Tech. Rep.18090781, 1995.

[5] Fang-Wei Fu, San Ling, and Chaoping Xing. New Lower Bounds and Constructions for Binary Codes Correcting Asymmetric Errors. In IEEE Trans. on Inform. Theory, volume 49, pages 3294-3299, March 2003.

[6] Amir Bennatan and David Burshtein. On the Application of LDPC Codes to Arbitrary Discrete-Memoryless Channels. In IEEE Trans. on Inform. Theory, volume 50, pages 417-438, March 2004.

[7] Edward A. Ratzer and David J.C. MacKay. Sparse Low-Density ParityCheck Codes for Channels with Cross-Talk. In Proc. ITW 2003, Paris, France, April 2003.

[8] G. Ungerboeck. Channel Coding with Multilevel Phase Signals. In IEEE Trans. on Inform. Theory, volume 28, pages 52-67, 1982.

[9] P. Ellingsen, S. Spinsante, and O. Ytrehus. Maximum Likelihood Decoding of Codes on the Asymmetric Z-channel. In the 10th IMA International Conference on Cryptography and Coding 2005.

[10] A. J. Viterbi. Convolutional codes and their performance in communication systems. IEEE Trans. on Comm., 19, Oct 1971.

[11] E. Biglieri. High-Level Modulation and Coding for Nonlinear Satellite Channels. IEEE Trans. on Comm., volume 32, May 1984.

[12] Y. J. Liu, I. Oka, and E. Biglieri. Error probability for digital transmission over non-linear channels with application to TCM. In IEEE Trans. on Inform. Theory volume 36, Sep 1990.

[13] M. Griot, A. I. Vila Casado, and R. Wesel. Non-linear Turbo Codes for Interleaver-Division Multiple Access on the OR Channel. Submitted to IEEE GLOBECOM Tech. Conf., Nov. 2006. 